\title{
The torsion generating set of the mapping class groups and the Dehn twist subgroups of non-orientable surfaces of odd genus
}

\author{
Xiaoming DU \\ (Received November 19, 2018) \\ (Revised February 1, 2020)
}

\begin{abstract}
Let $N_{g}$ be the non-orientable surface of genus $g$, $\operatorname{MCG}\left(N_{g}\right)$ the mapping class group of $N_{g}, \mathscr{T}\left(N_{g}\right)$ the index 2 subgroup generated by all Dehn twists of $\operatorname{MCG}\left(N_{g}\right)$. We prove that for odd genus, (1) if $g=4 k+3(k \geq 1), \operatorname{MCG}\left(N_{g}\right)$ can be generated by three elements of finite order; (2) if $g=4 k+1 \quad(k \geq 2), \mathscr{T}\left(N_{g}\right)$ can be generated by three elements of finite order.
\end{abstract}

\section{Introduction}

Let $N_{g}$ be the closed non-orientable surface of genus $g$. We denote by $\operatorname{Homeo}\left(N_{g}\right)$ the group consisting of all self-homeomorphisms of $N_{g}$, and by Homeo $_{0}\left(N_{g}\right)$ the normal subgroup consisting of homeomorphisms which are isotopic to the identity. Then the quotient group $\operatorname{Homeo}\left(N_{g}\right) / \operatorname{Homeo}_{0}\left(N_{g}\right)$ is called the mapping class group of $N_{g}$ and is denoted by $\operatorname{MCG}\left(N_{g}\right)$. The subgroup of $\mathrm{MCG}\left(N_{g}\right)$ generated by all Dehn twists is denoted by $\mathscr{T}\left(N_{g}\right)$.

Lickorish is the first one who discovered that $\mathscr{T}\left(N_{g}\right)$ is an index 2 subgroup of $\operatorname{MCG}\left(N_{g}\right)([6,7])$. Outside $\mathscr{T}\left(N_{g}\right)$, there is a mapping class called a "Y-homeomorphism" or a "crosscap slide". Chillingworth in [2] gave a finite set of generators for $\mathscr{T}\left(N_{g}\right)$ and hence also a finite set of generators for $\operatorname{MCG}\left(N_{g}\right)$. When the genus $g$ is low, for example, $g=2$, Lickorish found $\operatorname{MCG}\left(N_{2}\right) \cong \mathbb{Z}_{2} \oplus \mathbb{Z}_{2}$ and Chillingworth found $\mathscr{T}\left(N_{2}\right)$ can be generated by one Dehn twist $([6,2])$. When $g=3$, Birman and Chillingworth gave a concrete presentation for $\operatorname{MCG}\left(N_{3}\right)$ and then proved that $\operatorname{MCG}\left(N_{3}\right)$ can be generated by three elements $([1])$. Chillingworth found $\mathscr{T}\left(N_{3}\right)$ can be generated by two Dehn twists ([2]), and Szepietowski simplified Birman and Chillingworth's generating set into a set consisting of three involutions ([10]).

It is a natural question to what extent we can simplify the generating sets for $\operatorname{MCG}\left(N_{g}\right)$ and $\mathscr{T}\left(N_{g}\right)$ when $g$ is large. We would like to reduce both

The author is supported by the Fundamental Research Funds for the Central Universities in China and NSFC (Grant No. 11401219).

2010 Mathematics Subject Classification. 57N05, 57M20, $20 \mathrm{~F} 38$.

Key words and phrases. mapping class group, non-orientable surface, generator, torsion. 
the number and the orders of the generators. When $g \geq 4$, a generating set for $\operatorname{MCG}\left(N_{g}\right)$ consisting of four involutions was constructed by Szepietowski. Szepietowski also proved when $g \geq 4, \operatorname{MCG}\left(N_{g}\right)$ can be generated by three elements (see [10]). The first homology of $\operatorname{MCG}\left(N_{g}\right)$ has been calculated by Korkmaz [5]. As a consequence, Korkmaz proved that when $g=4$, the minimal number of the generators for $\operatorname{MCG}\left(N_{4}\right)$ is 3 . About $\mathscr{T}\left(N_{g}\right)$, Stukow gave a finite presentation of $\mathscr{T}\left(N_{g}\right)$ in [9]. Omori reduced the number of Dehn twist generators for $\mathscr{T}\left(N_{g}\right)$ to $g+1$ when $g \geq 4$ ([8]).

In [3], the author proved the following: when the genus $g^{\prime} \geq 5$ and $S_{g^{\prime}}$ is an orientable closed surface of genus $g^{\prime}$, the extended mapping class group $\mathrm{MCG}^{ \pm}\left(S_{g^{\prime}}\right)$ can be generated by two elements of finite order. One is of order 2 and the other is of order $4 g^{\prime}+2$. In the preprint [4], the author proved that the above result is also true for $g^{\prime}=3,4$. We found that the method in $[3,4]$ can be used in some of the cases of $\operatorname{MCG}\left(N_{g}\right)$ 's and $\mathscr{T}\left(N_{g}\right)$ 's. We have the following result:

THEOREM 1. Let $N_{g}, \operatorname{MCG}\left(N_{g}\right), \mathscr{T}\left(N_{g}\right)$ be as above.

(1) If $g=4 k+3(k \geq 1)$ (i.e. $g=7,11,15 \ldots), \operatorname{MCG}\left(N_{g}\right)$ can be generated by three elements of finite order. In the generating set, one of the generators is of order $2 g$, and the other two are of order 2.

(2) If $g=4 k+1(k \geq 2)$ (i.e. $g=9,13,17 \ldots), \mathscr{T}\left(N_{g}\right)$ can be generated by three elements of finite order. In the generating set, one of the generators is of order $2 g$, and the other two are of order 2.

\section{Preliminary}

\section{Crosscap slide.}

In $[6,7]$, Lickorish proved that $\left[\operatorname{MCG}\left(N_{g}\right): \mathscr{T}\left(N_{g}\right)\right]=2 . \quad$ As an example of the mapping classes which do not lie in $\mathscr{T}\left(N_{g}\right)$, he described a mapping class so-called a "Y-homeomorphism" or a "crosscap slide" as shown in Figure 1.
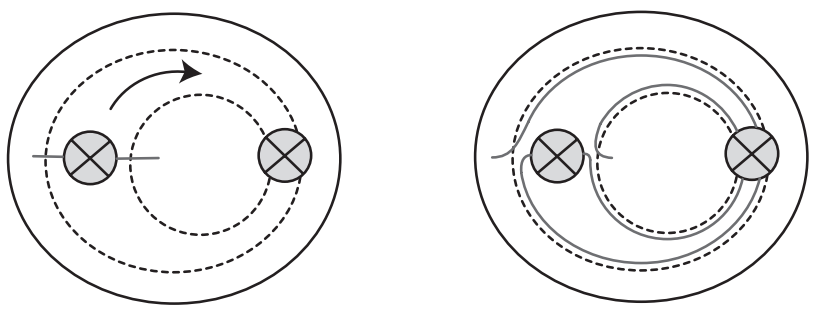

Fig. 1 

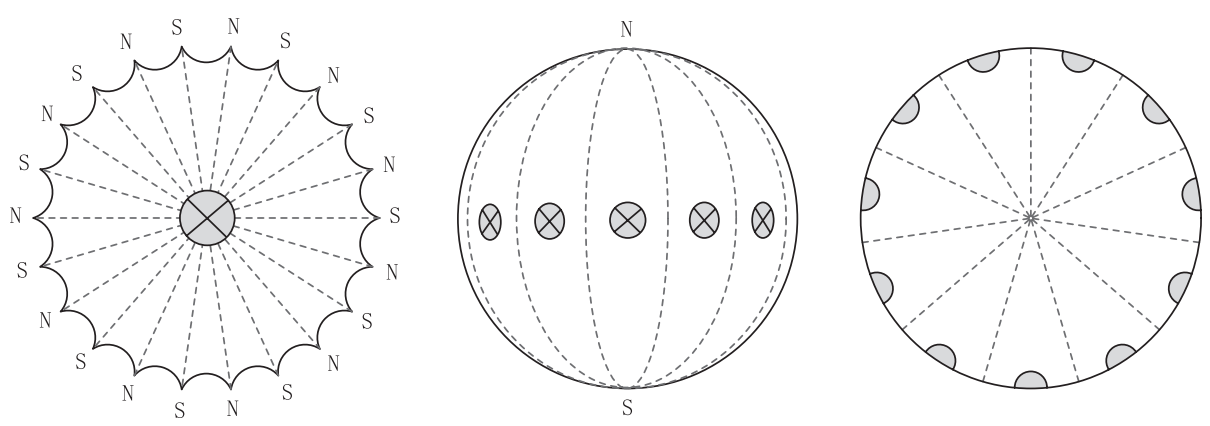

Fig. 2

Two points of view for the Möbius band partition of a non-orientable surface of odd genus.

If $g$ is odd, we can decompose the non-orientable surface $N_{g}$ into $g$ Möbius bands. Figure 2 shows two points of view to do this.

(1) The left picture of Figure 2 is a $2 g$-gon with a crosscap in the middle, and the opposite sides glued together pairwise. Under this gluing, the vertices of this $2 g$-gon are divided into two equivalence classes. After the gluing, they form two points on $N_{g}$. We denote them by $N$ and $S$. There are $g$ arcs in dotted lines connecting pairs of antipodal vertices and passing through the crosscap in the middle of the $2 g$-gon. They cut the $2 g$-gon into $g$ strips. After the gluing of the opposite sides of the $2 g$-gon, they form $g$ Möbius bands. We call it the $2 g$-gon presentation of $N_{g}$.

(2) The middle and the right pictures of Figure 2 show a 2-sphere with $g$ crosscaps. This is also $N_{g}$. Suppose the $g$ crosscaps sit on the equator. Denote the north pole and the south pole by $N$ and $S$, respectively. There are $g$ arcs in dotted lines connecting $N$ and $S$. They cut $N_{g}$ into $g$ Möbius bands. We call it the $g$-crosscap presentation of $N_{g}$.

We can check the above two presentations of $N_{g}$ are equivalent. In fact, in both presentations, we cut $N_{g}$ into $g$ Möbius bands. The points $N$ and $S$ are on the boundaries of these Möbius bands. We can build a homeomorphism on each Möbius band and then glue them together to make a global homeomorphism between the $2 g$-gon presentation of the surface and the $g$-crosscap presentation of the surface. In the following, we will go back and forth between the two presentations.

\section{Notations.}

(a) We use the convention of functional notation, namely, elements of the mapping class group are applied right to left, i.e. the composition $F G$ means that $G$ is applied first. 
(b) On an orientable surface, for each explicit two-sided simple closed curve, a Dehn twist means a right-handed Dehn twist along such a curve, and a left-handed Dehn twist is the inverse of a right-handed Dehn twist. On a non-orientable surface of odd genus, such as the left picture of Figure 2, we can cut off the crosscap in the middle of the $2 g$-gon presentation to get an orientable subsurface. So for each simple closed curve which is disjoint from the crosscap in the middle of the $2 g$-gon presentation, we can still define the right-handed Dehn twist in the oriented subsurface.

(c) We denote the curves by lower-case letters $a, b, c, d$ (possibly with subscripts) and the Dehn twists about them by the corresponding capital letters $A, B, C, D$. Notationally we do not distinguish a diffeomorphism/curve and its isotopy class.

The curves needed for generating $\mathscr{T}\left(N_{g}\right)$.

Omori constructed a generating set which consists of $g+1$ Dehn twists for $\mathscr{T}\left(N_{g}\right)$ ([8]). When we use the $g$-crosscap presentation of $N_{g}$, the curves for those Dehn twists are $a_{1}, a_{2}, \ldots, a_{g-1}, b_{0}, e$ shown in Figure 3 . We can check that a Dehn twist along $a_{1}$ maps $e$ to the curve $c$ in Figure 3. Hence the Dehn twists along $a_{1}, a_{2}, \ldots, a_{g-1}, b_{0}, c$ can also generate $\mathscr{T}\left(N_{g}\right)$.

We can also use the $2 g$-gon presentation to see what these curves are. See Figure 4.

We illustrate the verification of the correspondence of such curves as follows. The curves $a_{1}, a_{2}, \ldots, a_{g-1}$ form a chain of curves on $N_{g}$. Here a chain of curves means a set of curves $a_{1}, a_{2}, \ldots, a_{g-1}$ satisfying the following geometric intersection number conditions: (1) $i\left(a_{j}, a_{j+1}\right)=1(j=1,2, \ldots$, $g-1)$; (2) $i\left(a_{j}, a_{k}\right)=0(|j-k|>1)$. If we cut $N_{g}$ along $a_{1}, a_{2}, \ldots, a_{g-1}$, we can check that $N_{g}-\bigcup_{j=1}^{g-1} a_{j}$ is a Möbius band or a disk with a crosscap in the middle. The boundary of $N_{g}-\bigcup_{j=1}^{g-1} a_{j}$ consists of subarcs of $a_{j}$ 's. Each
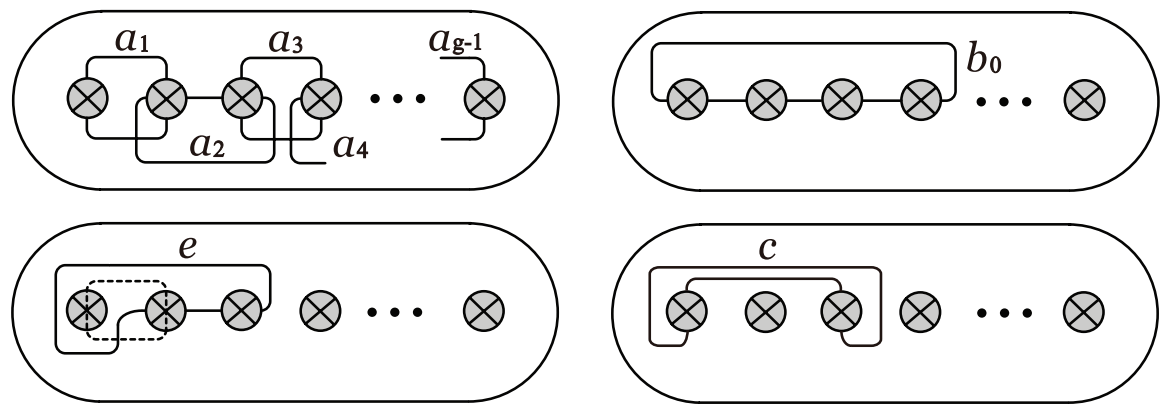

Fig. 3 

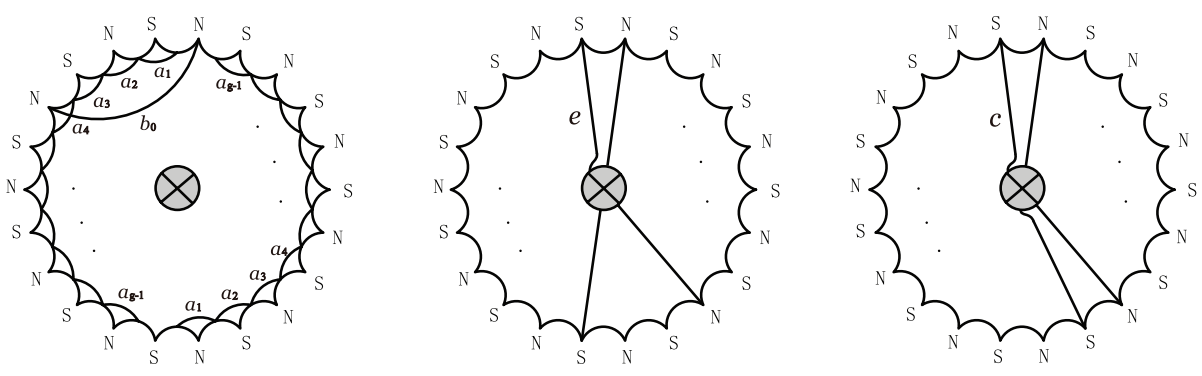

Fig. 4

two-sided curve $\gamma$ on $N_{g}$ will be cut into a union of arcs on $N_{g}-\bigcup_{j=1}^{g-1} a_{j}$. The end points of these arcs lie on the boundary of $N_{g}-\bigcup_{j=1}^{g-1} a_{j}$. These end points correspond to the intersection points of $\gamma$ with $a_{j}$ 's. Each arc on $N_{g}-\bigcup_{j=1}^{g-1} a_{j}$ is determined by its end points on the boundary and its relative position with the crosscap in the middle of the disk. Hence we can detect $\gamma$ by its intersection points with $a_{j}$ 's and the resulting arcs on $N_{g}-\bigcup_{j=1}^{g-1} a_{j}$. This gives the correspondence of the curves in both presentations of the nonorientable surface.

\section{The proof of the main theorem}

We now give the proof of Theorem 1.1.

Proof (Proof of Theorem 1.1). We first give the torsion generators. Suppose $g$ is odd. See Figure 5. Let $\sigma$ be the rotation of the $2 g$-gon presentation, $\tau_{1}$ the reflection of the $2 g$-gon presentation that preserves the curve $b_{0}$, and $\tau_{2}$ the reflection of the $g$-crosscap presentation that preserves $c$. We can easily see that $\left(\tau_{1} \circ B_{0}\right)^{2}=1,\left(\tau_{2} \circ C\right)^{2}=1, \sigma^{2 g}=1$.
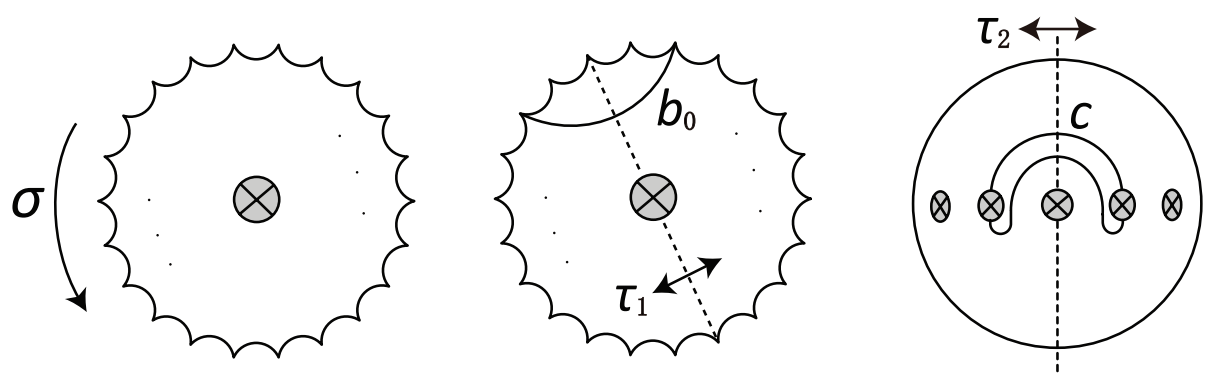

Fig. 5 
Let $G=\left\langle\sigma, \tau_{1} \circ B_{0}, \tau_{2} \circ C\right\rangle$ be the subgroup of $\operatorname{MCG}\left(N_{g}\right)$ generated by these three elements of finite orders. We will prove that: (1) if $g=7,11$, $15 \ldots$, then $G=\operatorname{MCG}\left(N_{g}\right)$; (2) if $g=9,13,17 \ldots$, then $G=\mathscr{T}\left(N_{g}\right)$.

The proof is by the following steps:

Step 1. Under the given conditions, we prove $G$ includes $A_{1}, \ldots$, $A_{g-1}, B_{0}, \tau_{1}$, and $\sigma$. Here $A_{1}, \ldots, A_{g-1}$, and $B_{0}$ are the Dehn twists along the curves $a_{1}, \ldots, a_{g-1}$, and $b_{0}$, respectively. They are shown in Figure 3 and 4 .

Step 2. We check $\tau_{2}$ is conjugate to $\tau_{1}$ by some power of $\sigma$ and then $\tau_{2}$ is in $G$. Hence $C$ is also in $G$. Here $C$ is the Dehn twist along the curve $c$ shown in Figure 3 and 4.

Step 3. By Omori's result [8], the fact that $A_{1}, \ldots, A_{g-1}, B_{0}, C$ are in $G$ implies $G$ includes $\mathscr{T}\left(N_{g}\right)$. Recall that $\left[\operatorname{MCG}\left(N_{g}\right): \mathscr{T}\left(N_{g}\right)\right]=2$. Hence $G$ is either $\mathscr{T}\left(N_{g}\right)$ or $\operatorname{MCG}\left(N_{g}\right)$.

Step 4. We check whether $\tau_{1}$ lies in $\mathscr{T}\left(N_{g}\right)$. If $\tau_{1}$ lies in $\mathscr{T}\left(N_{g}\right)$, then all the generators of $G$ is in $\mathscr{T}\left(N_{g}\right)$. Hence $G=\mathscr{T}\left(N_{g}\right)$. If $\tau_{1}$ does not lie in $\mathscr{T}\left(N_{g}\right)$, then $G=\operatorname{MCG}\left(N_{g}\right)$.

The proof of Step 1:

Take the $2 g$-gon presentation of $N_{g}$ ( $g$ is odd). If we remove the crosscap in the middle, then we get an orientable surface with genus $\frac{g-1}{2}$. In [3] and [4], for orientable surfaces, using the $2 g$-gon presentation, we generate $\operatorname{MCG}^{ \pm}\left(S_{(g-1) / 2}\right)$ by $\sigma$ and $\tau_{1} \circ B_{0}$ when $\frac{g-1}{2} \geq 3$. Here for the nonorientable surfaces, the method is similar. All the curves in the proof will not pass through the crosscap in the middle of the $2 g$-gon. In the following, we illustrate the main idea. For details, see [3] and [4]. We use the lantern relation $A B C D=X Y Z$, where $a, b, c, d, x, y, z$ are the curves on a 4-holed sphere. The lantern relation can also be written as $D=$ $\left(X A^{-1}\right)\left(Y B^{-1}\right)\left(Z C^{-1}\right)$. So one Dehn twist can be decomposed into a product of three pairs. Each pair consists of a left-handed Dehn twist and a right-handed Dehn twist. If we denote $b_{k}=\sigma^{k}\left(b_{0}\right)$, then we can see $\sigma^{k}\left(\tau_{1} \circ B_{0}\right) \sigma^{k}\left(\tau_{1} \circ B_{0}\right)=B_{k}^{-1} B_{0}$. Hence from $\sigma$ and $\tau_{1} \circ B_{0}$, we can get a pair, which consists of a left-handed Dehn twist and a right-handed Dehn twist. Conjugate such a pair by elements in $G$, we get many similar pairs, which include the three pairs $X A^{-1}, Y B^{-1}$, and $Z C^{-1}$ in the lantern relation. So there is at least one Dehn twist in $G$. We can also check such a Dehn twist can be chosen to be some $A_{j}$ or $B_{k}$. All $a_{j}$ 's are in the same $\sigma$-orbit. So every $A_{j}$ is in $G$. Similar for $B_{k}$ 's. The elements $\tau_{1} \circ B_{0}$ and $B_{0}$ are in $G$, so $\tau_{1}$ is in $G$. The neighbourhood of $\bigcup_{j=1}^{g-1} a_{j}$ is a one-holed orientable surface of genus $\frac{g-1}{2}$. By the chain relation, $\left(A_{g-1} A_{g-2} \ldots A_{1}\right)^{2 g}$ is a Dehn twist along the boundary curve of such a one-holed orientable surface. Such a curve bounds the crosscap in the middle of the $2 g$-gon presentation of $N_{g}$. 

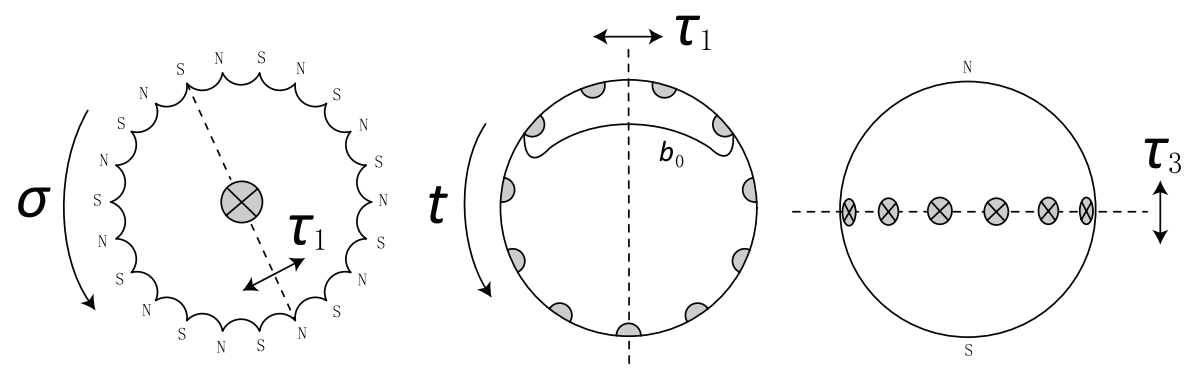

Fig. 6

The Dehn twist along such a curve is trivial. Hence $A_{g-1} A_{g-2} \ldots A_{1}$ equals the rotation $\sigma^{-1}$, and so $\sigma$ is in $G$.

The proof of Step 2:

We can interpret some of the torsion elements in more geometric ways. See Figure 6. We can check that $\tau_{1}$ is not only a reflection in the $2 g$-gon presentation but also a reflection in the $g$-crosscap presentation. Let $\tau_{3}$ be the north-south reflection of the $g$-crosscap presentation of $N_{g}, t$ be the order $g$ rotation. Since $\sigma$ gives a permutation of the $g$ Möbius bands and interchanges $N$ and $S$, we can see $\sigma=t \circ \tau_{3}$ and $\tau_{3}=\sigma^{g}$. Hence $\tau_{3}$ and $t$ are also in $G$. Now $\tau_{2}$ is conjugated to $\tau_{1}$ by some power of $t$. So $\tau_{2}$ also lies in $G$. Hence $C$ lies in $G$.

The proof of Step 3 is trivial.

The proof of Step 4:

In [7], Lickorish gave the following result: for a mapping class $f$ in $\operatorname{MCG}\left(N_{g}\right)$ and its induced automorphism $f_{*}$ on the $\mathbb{R}$-coefficient homology group $H_{1}\left(N_{g} ; \mathbb{R}\right)$, the element $f$ lies in $\mathscr{T}\left(N_{g}\right)$ (resp. does not lies in $\mathscr{T}\left(N_{g}\right)$ ) if and only if $f_{*}$ has determinant +1 (resp. -1 ). In the $g$-crosscap presentation of $N_{g}$, take $g$ one-sided simple closed curves which are the core curves of the $g$ crosscaps. Since $\tau_{1}$ is a reflection of the $g$-crosscap presentation, it exchanges $g-1$ core curves pairwise and reverse their orientations. These $g-1$ core curves form a basis for $H_{1}\left(N_{g} ; \mathbb{R}\right)$. The induced automorphism $\left(\tau_{1}\right)_{*}$ of $H_{1}\left(N_{g} ; \mathbb{R}\right)$ with respect to such a basis gives a $(g-1) \times(g-1)$ matrix

$$
\left(\begin{array}{ccccc}
0 & 0 & \ldots & 0 & -1 \\
0 & 0 & \ldots & -1 & 0 \\
\vdots & \vdots & \vdots & \vdots & \vdots \\
0 & -1 & \ldots & 0 & 0 \\
-1 & 0 & \ldots & 0 & 0
\end{array}\right)
$$


When $g=4 k+3$, the determinant is $-1, \tau_{1}$ does not lie in $\mathscr{T}\left(N_{g}\right)$. When $g=4 k+1$, the determinant is $+1, \tau_{1}$ lies in $\mathscr{T}\left(N_{g}\right)$. This completes the proof.

\title{
Acknowledgement
}

The author would like to thank Szepietowski for telling many results and pointing out some generator was missing in the earlier version of the paper. The author is very grateful to the referee for suggestions to improve many statement and pointing out a mistake in the original submitted paper.

\section{References}

[1] J. S. Birman and D. R. J. Chillingworth, On the homeotopy group of a non-orientable surface. Proc. Cambridge Philos. Soc. 71 (1972), 437-448.

[2] D. R. J. Chillingworth, A finite set of generators for the homeotopy group of a nonorientable surface. Proc. Cambridge Philos. Soc. 65 (1969), 409-430.

[3] X. Du, The extended mapping class group can be generated by two torsions. J. Knot Theory Ramifications, Volume No. 26 (2017), Issue No. 11.

[4] X. Du, The torsion generators of the extended mapping class groups in low genus cases, preprint.

[5] M. Korkmaz, First homology group of mapping class groups of non-orientable surfaces. Proc. Cambridge Philos. Soc. 123 no. 3 (1998), 487-499.

[6] W. B. R. Lickorish, Homeomorphisms of non-orientable two-manifolds. Proc. Cambridge Philos. Soc. 59 (1963), 307-317.

[7] W. B. R. Lickorish, On the homeomorphisms of a non-orientable surface. Proc. Cambridge Philos. Soc. 61 (1965), 61-64.

[8] G. Omori, A small generating set for the twist subgroup of the mapping class group of a non-orientable surface by Dehn twists. Hiroshima Math. J. Volume 48, Number 1 (2018), 81-88.

[9] M. Stukow, A finite presentation for the twist subgroup of the mapping class group of a non-orientable surface, Bull. Korean Math. Soc. 53 (2016), no. 2, 601-614.

[10] B. Szepietowski, The mapping class group of a non-orientable surface is generated by three elements and by four involutions. Geom. Dedicata 117 (2006), 1-9.

\author{
Xiaoming $D u$ \\ School of Mathematics \\ South China University of Technology \\ Guangzhou 510640, P.R. China \\ E-mail: scxmdu@scut.edu.cn
}

